\title{
UM ENSAIO SOBRE A HISTÓRIA DA TRIGONOMETRIA ANTES DO SÉCULO XV
}

\author{
Ana Carolina Costa Pereira ${ }^{1}$, Bernadete Barbosa Morey ${ }^{2}$ \\ ${ }^{1}$ Universidade Estadual do Ceará \\ ${ }^{2}$ Universidade Federal do Rio Grande do Norte \\ <carolina.pereira@uece.br>, <bernadetemorey@gmail.com> \\ DOI: 10.21439/conexoes.v9i4.933
}

\begin{abstract}
Resumo. Neste artigo apresentamos um breve percurso pela história da Trigonometria, relembrando alguns fatos importantes desde seu nascimento, como uma ciência voltada para resolver problemas na Astronomia, até antes de sua emancipação, como uma ciência independente nos séculos XV a XVII. Nesse percurso, inicialmente discutimos o nascimento da Trigonometria como ciência auxiliar da Astronomia; a Trigonometria das Cordas com Ptolomeu e o seu Almagesto; a Trigonometria indiana das semi-cordas; a Trigonometria Árabe e a expansão da Trigonometria em precisão das tabelas e no aumento das funções trigonométricas. Finalmente, expomos sobre o surgimento da Trigonometria como uma ciência independente, na Europa dos séculos XV a XVII.
\end{abstract}

Palavras-chaves: História da Trigonometria. Astronomia. Trigonometria da corda e da semi-corda. Tabelas trigonométricas.

\begin{abstract}
This article presents a brief journey through the history of trigonometry, remembering some important facts from its birth, as a science focused on solving problems in astronomy, even before its emancipation, as an independent science on the fifteenth to seventeenth centuries. Along the way, initially we discussed the birth of trigonometry as auxiliary science of Astronomy; the String Trigonometry with Ptolemy and his Almagest; the Indian trigonometry of half-chords; the Arabic trigonometry and the expansion of Trigonometry in precision of the tables and the increase of the trigonometric functions. Finally, we talked about the emergence of trigonometry as an independent science in Europe of the fifteenth to seventeenth centuries.
\end{abstract}

Keywords: History of Trigonometry. Astronomy. Trigonometry rope and half-chords. Trigonometric table.

\section{O NASCIMENTO DA TRIGONOMETRIA COMO CIÊNCIA AUXILIAR DA ASTRONO- MIA}

A Trigonometria, hoje, um dos ramos da Matemática, surgiu e desenvolveu-se como ferramenta cuja finalidade era auxiliar a Astronomia, ainda na Antiguidade. A relação entre essas duas áreas era tão intrínseca que se tornou proveitoso considerar sua separação somente na Idade Média. Na verdade, o primeiro tratado de Trigonometria independente da Astronomia de que temos

${ }^{1}$ Esse artigo é uma adaptação de um dos capítulos da tese: A Obra "De Triangulis Omnimodis Libri Quinque" de Johann Muller Regiomontanus (1436 - 1476): uma contribuição para o desenvolvimento da Trigonometria (PEREIRA 2010). notícia foi o Tratado dos Quadriláteros, de Nasīr alDīn al-Tūsī, no século XIII. Já na Europa, a Trigonometria foi abordada de modo independente da Astronomia pela primeira vez no De triangulis omnimodis libri quinque, de Regiomontanus, obra escrita por volta de 1464 e publicada postumamente em 1533.

Autores como Zeller (1944) e Zinner (1990) discordam na questão de Regiomontanus ter tido ou não acesso à obra de $a l-T \bar{u} s \bar{t}^{2}$ Deste modo, nada podemos inferir sobre a influência da obra de $a l-T \bar{u} s \bar{\imath}$ no desen-

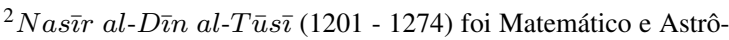
nomo árabe. Contribuiu bastante para a Trigonometria plana e esférica e a Astronomia, onde suas conclusões foram, inclusive, utilizadas por Copérnico. Escreveu o primeiro trabalho sobre Trigonometria que a trata independente da Astronomia (ZELLER $\mid$ 1944). 
UM ENSAIO SOBRE A HISTÓRIA DA TRIGONOMETRIA ANTES DO SÉCULO XV, CEARÁ, BRASIL.

volvimento da Trigonometria na Europa.

\section{A TRIGONOMETRIA DAS CORDAS}

Dentre as primeiras contribuições para a Trigonometria de que temos notícias, estão as contribuições dos babilônicos advindas de suas observações astronômicas 3 e registradas em suas inúmeras tábuas. Uma tábua babilônica famosa é a chamada Plimpton 322 cujo conteúdo, segundo as mais recentes interpretações, referese aos valores da cossecante de ângulos de $31^{\circ}$ a $45^{\circ}$. Rudimentos de Trigonometria aparecem ainda no $\mathrm{Pa}$ piro de Rhind ou Papiro de Ahmes, ( 1650 a.C.) o que demonstra que os egípcios já possuíam instrumentos matemáticos para medir inclinação.

Os problemas 56 a 60 do Papiro de Rhind contêm cálculos sobre a inclinação da Pirâmide relacionando o seqed. Uma interpretação de seked seria o cosseno do ângulo que é formado pela margem lateral com a diagonal da base, e uma segunda interpretação seria a cotangente do ângulo.

No entanto, foi na Grécia do século III a.C. que a Trigonometria adquiriu uma sistematização a partir da Geometria, segundo o modelo grego de raciocínio lógico dedutivo, com Hiparco de Nicéia 4 ( 190 a.C. 126 a.C.) e Claudius Ptolomed ${ }^{5}(\sim 87$ a.C $\sim \sim 150$ a.C $)$ com sua Trigonometria das cordas.

Embora não tenhamos certeza, por volta de 150 a.C., Hiparco teria escrito uma obra em doze livros sobre o cálculo de comprimento de cordas, na qual estava incluída a primeira tabela trigonométrica baseada em uma função: a corda de um arco de círculo arbitrário. Infelizmente, essa obra não chegou até nós. Consta ainda que Hiparco fez o cálculo da distância Terra-Lua a partir da simples contagem do tempo de um eclipse lunar. Para fazer este cálculo, ele utilizou tabelas trigonométricas talvez de origens babilônicas. Brummelen (2009, p. 44) tenta fazer uma reconstituição da tabela de cordas de Hiparco (Tabela 1).

Acreditamos que a obra de Hiparco tenha servido de base para a obra que se tornou um marco para o estudo da Astronomia e na qual aparece a primeira tabela trigonométrica que chegou até nós. Estamos falando do Almagesto, do astrônomo Claudius Ptolomeu, em torno de 150 a.C.

Escrito com o título Matematike Syntaxis ou Composição Matemática, tornou-se mais conhecido por seu

\footnotetext{
${ }^{3}$ Ver Neugebauer (1969).

${ }^{4}$ Hiparco de Nicéia é considerado o pai da Trigonometria por ter sido o pioneiro na elaboração de uma tabela trigonométrica, com valores de arcos e cordas para uma série de ângulos e o fundador da Astronomia científica.

${ }^{5}$ Claudius Ptolomeu ( 100 d.C. $\mathrm{a} \sim 170$ d.C. $)$ se dedicou às ciências matemáticas, em especial à Astronomia.
}

Tabela 1: Reconstituição da tabela de cordas de Hiparco.

\begin{tabular}{|c|c|}
\hline$\theta$ & $\operatorname{Crd} \theta$ \\
\hline 0 & 0 \\
\hline $71 / 2^{\circ}$ & 450 \\
\hline $15^{o}$ & 897 \\
\hline $221 / 2^{\circ}$ & 1341 \\
\hline $30^{\circ}$ & 1780 \\
\hline $371 / 2^{\circ}$ & 2210 \\
\hline$\ldots$ & $\ldots$ \\
\hline $180^{\circ}$ & 6875 \\
\hline
\end{tabular}

nome de influência árabe, o Almagesto. Foi traduzido do grego várias vezes, primeiro para o siríaco e depois para o árabe. A tradução do árabe para o latim, feita por Gerard de Cremona, em Toledo, no século XII, foi possibilitando a difusão do conhecimento do Almagesto na Europa.

Esta obra de Ptolomeu é exclusivamente concernente a uma descrição de métodos unificados para a representação dos fenômenos celestes (MOREY, 2001). Em síntese, é a exposição completa da Astronomia matemática, de acordo com o entendimento grego do termo. Segundo Asger (1984, p. 127), o

\footnotetext{
Almagesto desempenhou o mesmo papel na Astronomia matemática que os Elementos de Euclides e as Cônicas de Apolônio em seus respectivos assuntos. (...) Mas Ptolomeu, diferentemente de Euclides, reconheceu as realizações de seus antecessores generosa e precisamente, de maneira que nosso conhecimento da Astronomia préptolomaica é mais rico e mais firme do que o da matemática pré-euclidiana. Pela mesma razão podemos identificar bem as contribuições do próprio Ptolomeu.
}

O Almagesto foi escrito como uma coleção de treze livros que defendiam a visão cosmológica segundo a qual a Terra ficava no centro do universo, e todos os astros girando em torno dela. Ptolomeu supôs a Lua e os planetas em movimento uniforme sobre círculos chamados epiciclos (Figura 1). Por sua vez, o centro de um epiciclo estaria se movendo uniformemente ao longo de outro círculo maior chamado deferente.

A estrutura do Almagesto não é diferente das obras da época: ele começa com a matemática básica que será usada e mostra como obter as entradas na sua tabela trigonométrica. Começa com o cálculo da função trigonométrica da corda, aplicando-lhe uma longa série de demonstrações, construções e derivações dos parâmetros numéricos de seu material observacional (MOREY). 2001). Assim, no livro I, Ptolomeu constrói uma tabela trigonométrica, a ferramenta principal de suas descobertas astronômicas. No livro II, discute aspectos da 


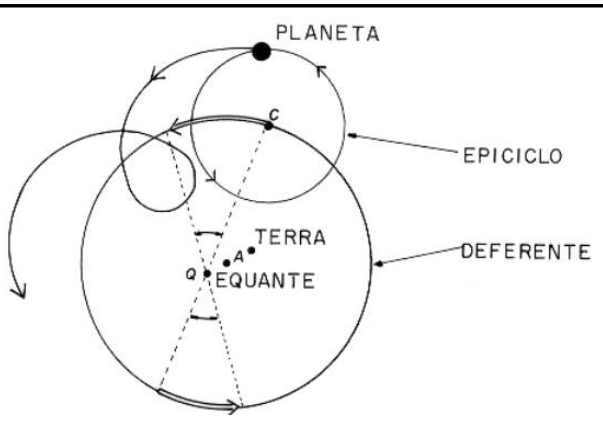

Figura 1: Sistema de epiciclos de Ptolomeu.

Fonte: Disponível em: <http://www.ghtc.usp.br/server/Sites-HF/ Geraldo/ptolomaico_arquivos/image002.gif $>$ Acesso em: $13 / 11 / 2015$.

Astronomia esférica do ponto de vista do observador da Terra. No Livro III, oferece a Teoria do Sol. No Livro IV, discute a Teoria da Lua. O Livro V é a continuação da teoria lunar e trata também das paralaxes solares e lunares. O Livro VI é dedicado às tábuas da Lua e dos eclipses. Os Livros VII e VIII tratam de estrelas fixas. Já os últimos cinco livros são dedicados aos planetas (ZINNER, 1990).

$$
\text { Segundo (MOREY, 2001, p. 30), }
$$

\footnotetext{
Ptolomeu não discute certos tópicos, seja porque ele os considera de conhecimento de todos os seus leitores, ou seja, porque ele considera supérfluo entrar em detalhes, tais como: Geometria (encontrada em Euclides), logística (como cálculo da raiz quadrada) e esférica. Na sua exposição Ptolomeu lança mão da geometria elementar, sistema de numeração de base sessenta, das frações gregas e, o que mais nos interessa, da Trigonometria.
}

Porém, percebemos alguns conhecimentos inseridos no Almagesto que não são creditados a Ptolomeu: a notação sexagesimal e a operação de multiplicação herdadas dos babilônicos e a divisão dos egípcios; extração da raiz quadrada e conhecimentos geométricos contidos nos Elementos de Euclides.

Dentre as tabelas trigonométricas da Antiguidade, a mais famosa é a tabela de cordas de Ptolomeu, inserida no Almagesto (MOREY, 2001). Na construção da tabela, Ptolomeu relaciona o comprimento do arco de uma circunferência com a medida em graus do arco/ângulo central correspondente.

Observando a Figura 2, podemos, por meio de raciocínio geométrico, estabelecer a equivalência entre o conceito de comprimento de corda de um ângulo central e o seno da metade deste mesmo ângulo. É com base em tal equivalência que dizemos que a tabela de cordas de Ptolomeu é uma tabela trigonométrica.

Ou seja $A O=O B=r=60$ partes. Como $A B$ é

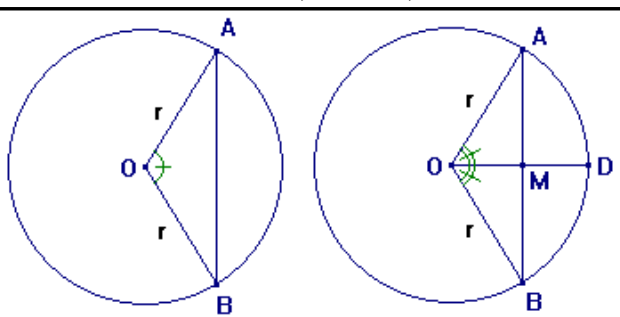

Figura 2: Equivalência entre seno e corda.

Fonte: Elaborada pelas autoras.

a corda $=\operatorname{crd} 2 \alpha, 2 \alpha=A \hat{\mathrm{O}} B, A \hat{\mathrm{O}} M=B \hat{\mathrm{O}} M=\alpha \mathrm{e}$ $O D \perp A B$, então $\operatorname{sen} \alpha=\frac{A M}{O A}=\frac{2 A M}{2 O A}=\frac{c r d 2 \alpha}{120}$.

Como unidade de comprimento de corda, Ptolomeu usou o que ele chamou de parte, ou seja, 1/120 do comprimento do diâmetro da circunferência. A medida usada para o arco foi o grau (1/360 da circunferência completa). Para expressar as subdivisões dos comprimentos das cordas, Ptolomeu lançou mão do sistema sexagesimal. Com estes recursos, ele calculou os valores das cordas para todo ângulo central de $0^{\circ}$ a $180^{\circ}$, em passos de $1 / 2^{\circ}$.

Para a construção dessas tabelas, Ptolomeu utilizou o cálculo das cordas de alguns ângulos, identificandoas por meio dos lados de polígonos regulares inscritos como, por exemplo: $\operatorname{crd} 36^{\circ}$ é o lado do decágono inscrito; $\operatorname{crd} d 60^{\circ}$ é o lado do hexágono inscrito; $\operatorname{crd} 72^{\circ}$ é o lado do pentágono inscrito; $\operatorname{cr} d 90^{\circ}$ é o lado do quadrado inscrito; $\operatorname{crd} 120^{\circ}$ é o lado do triângulo equilátero inscrito. Para isso, ele apresenta a construção desses polígonos e a determinação do comprimento do lado desses polígonos (Tabela 2).

Para o cálculo dos demais ângulos, Ptolomeu deduz, sempre geometricamente, e utiliza as fórmulas da corda da diferença de dois arcos, a corda do arco-metade e a corda da soma de dois arcos. Obtém então os seguintes resultados: 
Tabela 2: Tábua com cordas de alguns ângulos

\begin{tabular}{cc}
\hline Ângulo & Corda \\
\hline $36^{\circ}$ & $37^{p} 04^{\prime} 55^{\prime \prime}$ \\
$60^{\circ}$ & $60^{p}$ \\
$72^{\circ}$ & $70^{p} 32^{\prime} 03^{\prime \prime}$ \\
$90^{\circ}$ & $84^{p} 51^{\prime} 10^{\prime \prime}$ \\
$108^{\circ}$ & $97^{p} 04^{\prime} 56^{\prime \prime}$ \\
$120^{\circ}$ & $103^{p} 55^{\prime} 23^{\prime \prime}$ \\
$144^{\circ}$ & $114^{p} 07^{\prime} 37^{\prime \prime}$ \\
$180^{\circ}$ & $120^{p}$ \\
\hline
\end{tabular}

Fonte: Morey (2001, p. 36)

A corda da diferença de dois arcos: Sendo conhecidos os comprimentos de cordas de dois arcos, é possível encontrar a corda do arco que é a diferença dos dois primeiros.

$$
\operatorname{crd}(\Theta-\varphi)=\frac{\operatorname{crd} \sqrt{120^{2}-\operatorname{crd}^{2} \varphi}-\operatorname{crd} \varphi \sqrt{120^{2}-\operatorname{crd}^{2} \Theta}}{120}
$$

A corda do arco-metade: Sendo considerado o comprimento de corda de um arco, é possível encontrar a corda do arco que é a metade do arco dado.

$$
\operatorname{crd}\left(\frac{\Theta}{2}\right)=\sqrt{7200-60 \sqrt{120^{2}-\operatorname{crd}^{2} \Theta}}
$$

A corda da soma de dois arcos: Sendo conhecidos os comprimentos de cordas de dois arcos, é possível encontrar a corda do arco que é a soma dos dois primeiros.

$$
\operatorname{crd}(\Theta+\varphi)=\frac{\operatorname{crd} \sqrt{120^{2}-\operatorname{crd} d^{2} \varphi}+\operatorname{crd} \varphi \sqrt{120^{2}-c r d^{2} \Theta}}{120}
$$

Lançando mão dos comprimentos de cordas constantes da Tabela 1 e das fórmulas (1), (2) e (3), é possível encontrar os comprimentos de cordas dos arcos de $\left(3 / 2^{n}\right)$, para valores inteiros de $n$. No entanto, é impossível encontrar os comprimentos de corda para os arcos de $1^{\circ}$.

Ptolemy's apud Morey (2001) escreve algumas considerações:

É óbvio que combinando a corda $\left(1 \frac{1}{2}\right)^{\circ}$ com todas as cordas que já obtivemos, seremos capazes de calcular o comprimento de todas as cordas cujos arcos são múltiplos de $\left(1 \frac{1}{2}\right)^{\circ}$. E assim, as únicas cordas que faltam ser calculadas são aquelas entre os intervalos de $\left(1 \frac{1}{2}\right)^{\circ}$, duas em cada intervalo. Se então, tivéssemos o valor crd $\frac{1}{2}^{\circ}$ poderíamos preencher a tabela com as cordas faltantes utilizando a soma ou a diferença (da corda de $\frac{1}{2}^{\circ}$ ) com a corda de uma das extremidades de cada intervalo. No entanto, dada a corda de um arco, digamos, de $\left(1 \frac{1}{2}\right)^{\circ}$, a corda de um terço deste arco não pode ser encontrada por meio geométricos (problema da trissecção do ângulo). Deste modo obteremos a corda de $1^{\circ}$ a partir das cordas de $\left(1 \frac{1}{2}\right)^{\circ} \mathrm{e}\left(\frac{3}{4}\right)^{\circ}$. Faremos isto lançando mão de um lema que apesar de não permitir, de modo geral, calcular tamanho de cordas, permite, no caso de pequenas cordas, calcular seu comprimento com um erro tão pequeno quanto se queira.

Para encontrar a $\operatorname{crd} 1^{\circ}$ e deste modo completar a tabela, Ptolomeu recorreu a uma interpolação que permitiu deduzir a seguinte desigualdade: 


$$
0,01745130<\text { seno de } 1^{o}<0,01745279
$$

De posse da aproximação (4) acima, Ptolomeu pôde então finalizar sua tabela e fornecer os comprimentos de cordas para arcos de 0 a $180^{\circ}$ variando de $1 / 2 \mathrm{em} 1 / 2$ grau.

O Almagesto de Ptolomeu com sua tabela de cordas serviu de base para estudos em Astronomia, na Europa, durante vários séculos. O passo seguinte da evolução dos conceitos trigonométricos foi dado pelos indianos, com a introdução da semi-corda.

\section{A TRIGONOMETRIA DAS SEMI-CORDAS}

O que temos de registro sobre Trigonometria na Índia pode ser encontrado nos manuais de Astronomia, chamados Siddhāntas: Sūrya - Siddhānta ( 500 d.C.), Paca - Siddhāntikā ( 628 d.C.) e Brāhmasphuta - Siddhānta ( 628 d.C).

A obra Sürya-Siddhānta, de autor desconhecido, é um dos mais notáveis desses textos sobre Astronomia que sobreviveu aos nossos tempos. Ele era constituído de regras crípticas em versos redigidos em sânscrito, com poucas explicações e nenhuma prova (KENNEDY 1992).

No que se refere ao estudo das funções trigonométricas, os indianos utilizavam a meia-corda, que posteriormente seria o seno indiano. Segundo Morey (2003 p. 19-20),

\footnotetext{
[...] para os indianos as funções trigonométricas ainda eram definidas como comprimento de um segmento e não como uma relação entre dois comprimentos, como é o caso as funções trigonométricas modernas. Então quando dizemos seno indiano estamos nos referindo ao comprimento da meia-corda do ângulo central.
}

Observe a Figura 3 . Nela podemos encontrar os segmentos referentes ao seno indiano, ao cosseno indiano e ao seno reverso $(1-\cos \beta)$. Podemos estabelecer, a partir da Figura 3 , a relação entre as funções trigonométricas modernas e as indianas. De fato, seja $M \hat{\mathrm{O}} A=\beta$ e $A O=B O=r$ então

$A M=j y a \beta=r \cdot \operatorname{sen} \beta=($ seno indiano $)$

$O M=k o j y a \beta=r \cdot \cos \beta=($ cosseno indiano $)$

$M C=O C-O M=$ ukamajya $\beta=r-r \cdot \cos \beta=$ $r(1-\cos \beta)=\operatorname{vers} \beta$

Varahamihira (c. 505-587 d.C) ao calcular jya $\beta=$ $r \cdot \operatorname{sen} \beta$ para $\beta \leq 90^{\circ}$, sugeriu o uso das equações:

$$
\begin{aligned}
& \text { jya } 30^{\circ}=\frac{1}{2} \cdot r \\
& \text { jya } 60^{\circ}=\frac{1}{2} \cdot \sqrt{r}
\end{aligned}
$$

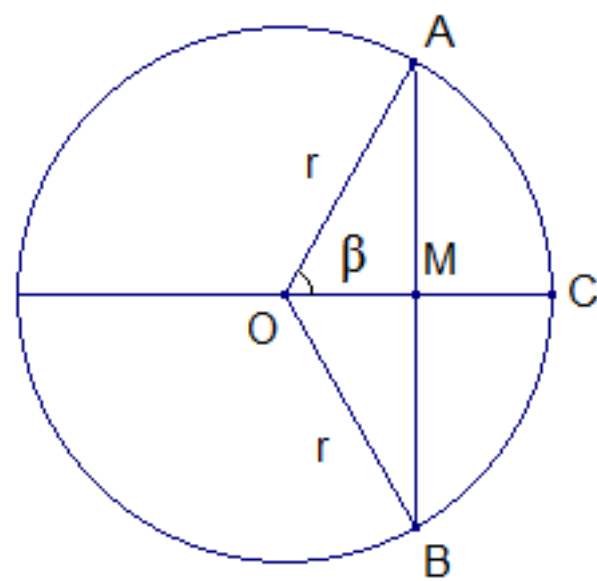

Figura 3: Seno indiano, Cosseno e Seno Reverso

Fonte: Elaborada pelas autoras.

$$
\text { jya } 90^{\circ}=r
$$

Partindo das equações anteriores, Varahamihira calculou valores de $j y a \beta(r \cdot \operatorname{sen} \beta)$ para todos os múltiplos de $3^{\circ} 45^{\prime}$ até $90^{\circ}$. Provavelmente, ele utilizou o equivalente das fórmulas

$$
\begin{gathered}
\operatorname{sen} x=\cos (\pi / 2-x) \\
\operatorname{sen}^{2} x+\cos ^{2} x=1
\end{gathered}
$$

$\operatorname{sen}^{2} x=1 / 4\left(\operatorname{sen}^{2} 2 x+\operatorname{ver} \operatorname{sen}^{2} 2 x=1 / 2(1-\cos 2 x)\right.$

e outras conhecidas na época, para encontrar o valor do ângulo $3^{\circ} 45^{\prime}$ por meio do sen $90^{\circ}$. Os valores encontrados são mostrados na Tabela 3 .

Outro modo utilizado pelos hindus para obter a tabela da Figura 3 era por meio da fórmula de recorrência (11), com $n$ variando de 1 a 23.

$$
\begin{gathered}
\operatorname{sen}(n+1) x-\operatorname{sen}(n x)= \\
\operatorname{sen}(n x)-\operatorname{sen}(n-1) x-\left(\frac{1}{255} \operatorname{sen}(n x)\right)
\end{gathered}
$$

Aryabhata escreveu um trabalho que é essencialmente a sistematização dos resultados contidos no Siddhāntas. Ele deu outras medidas de raios, permitindo assim que o seno fosse expresso em um tipo de medida circular

\section{A EXPANSÃO DA TRIGONOMETRIA EM PRECISÃO DAS TABELAS E NO AUMENTO DAS FUNÇÕES TRIGONOMÉTRICAS}

Os árabes proporcionaram um grande progresso à Matemática, e em particular, à Trigonometria, que poste- 
UM ENSAIO SOBRE A HISTÓRIA DA TRIGONOMETRIA ANTES DO SÉCULO XV, CEARÁ, BRASIL.

Tabela 3: Tabela de senos indianos calculada por Varahamihira, Aryabhata I e seno moderno.

\begin{tabular}{|c|c|c|c|c|c|}
\hline \multirow[b]{2}{*}{$\begin{array}{c}\text { Ângulo } \\
\Theta\end{array}$} & \multicolumn{2}{|c|}{ Varahamihira } & \multicolumn{2}{|c|}{ Aryabhata I } & \multirow{2}{*}{$\begin{array}{c}\text { Valor Moderno } \\
\operatorname{sen} \theta\end{array}$} \\
\hline & $\begin{array}{l}r \cdot \operatorname{sen} \theta \\
r=120\end{array}$ & $\operatorname{sen} \theta$ & $\begin{array}{l}r \cdot \operatorname{sen} \theta \\
r=3438\end{array}$ & $\operatorname{sen} \theta$ & \\
\hline $3^{\circ} 45^{\prime}$ & 751 & 0,06542 & $225^{\prime}$ & 0,6545 & 0,06540 \\
\hline $7^{\circ} 30^{\prime}$ & 1540 & 0,13056 & $449^{\prime}$ & 0,13060 & 0,13056 \\
\hline $11^{\circ} 15^{\prime}$ & 2325 & 0,19514 & 671' & 0,19517 & 0,19509 \\
\hline $15^{\circ}$ & 31 '4"' & 0,25889 & $890^{\prime}$ & 0,25962 & 0,25882 \\
\hline $18^{\circ} 45^{\prime}$ & $38^{\prime} 34^{\prime \prime}$ & 0,32139 & $1105^{\prime}$ & 0,32141 & 0,32143 \\
\hline $22^{\circ} 30^{\prime}$ & $45^{\prime} 56^{\prime \prime}$ & 0,38278 & $1315^{\prime}$ & 0,38249 & 0,38268 \\
\hline $26^{\circ} 15^{\prime}$ & $53^{\prime} 5^{\prime \prime}$ & 0,44236 & $1520^{\prime}$ & 0,44212 & 0,44229 \\
\hline $30^{\circ}$ & $60^{\prime}$ & 0,50000 & $1719^{\prime}$ & 0,50000 & 0,50000 \\
\hline $33^{\circ} 45^{\prime}$ & $66^{\prime} 40^{\prime \prime}$ & 0,55556 & 1910' & 0,55556 & 0,55556 \\
\hline $37^{\circ} 30^{\prime}$ & 73'3'" & 0,60875 & 2093' & 0,60878 & 0,60876 \\
\hline $41^{\circ} 15^{\prime}$ & 79'7'” & 0,65931 & $2267^{\prime}$ & 0,65910 & 0,65935 \\
\hline $45^{\circ}$ & $84 ' 51 "$ & 0,70708 & $2431^{\prime}$ & 0,70710 & 0,70711 \\
\hline $48^{\circ} 45^{\prime}$ & 90’13" & 0,75181 & $2585^{\prime}$ & 0,75189 & 0,75184 \\
\hline $52^{\circ} 30^{\prime}$ & 95'13" & 0,79347 & $2728^{\prime}$ & 0,79348 & 0,79335 \\
\hline $56^{\circ} 15^{\prime}$ & 99'46" & 0,83139 & $2859^{\prime}$ & 0,83159 & 0,83147 \\
\hline $60^{\circ}$ & $103 ' 56 "$ & 0,86611 & $2978^{\prime}$ & 0,86620 & 0,86602 \\
\hline $63^{\circ} 45^{\prime}$ & 107 '38" & 0,89694 & $3084 '$ & 0,89703 & 0,89687 \\
\hline $67^{\circ} 30^{\prime}$ & $110 ' 53 ”$ & 0,92402 & $3177^{\prime}$ & 0,92408 & 0,92388 \\
\hline $71^{\circ} 15^{\prime}$ & 113'38" & 0,94694 & $3256^{\prime}$ & 0,94706 & 0,94693 \\
\hline $75^{\circ}$ & $115 ' 56 "$ & 0,96611 & $3321^{\prime}$ & 0,96597 & 0,96593 \\
\hline $78^{\circ} 45^{\prime}$ & 117 '43" & 0,98097 & $3372^{\prime}$ & 0,98080 & 0,98079 \\
\hline $82^{\circ} 30^{\prime}$ & $119^{\prime}$ & 0,99167 & $3409^{\prime}$ & 0,99156 & 0,99144 \\
\hline $86^{\circ} 15^{\prime}$ & $119^{\prime} 45^{\prime \prime}$ & 0,99792 & $3431^{\prime}$ & 0,99796 & 0,99786 \\
\hline $90^{\circ}$ & $120^{\prime}$ & 1,00000 & $3438^{\prime}$ & 1,00000 & 1,00000 \\
\hline
\end{tabular}

Fonte: Morey (2003. p. 25)

riormente influenciou o desenvolvimento destas disciplinas na Europa Medieval e Renascentista. Com relação à Trigonometria, podemos citar, entre os mais importantes, os trabalhos de $A b \bar{u}^{\prime} l-W a f \bar{a}$ al $B \bar{u} z j \bar{a} n \bar{x}^{6}$ (940-998) e Nasīr al-Dīnal-Tūsì (1201-1274). Entretanto eles não conseguiram conquistar o reconhecimento por quase três séculos.

$\mathrm{Na}$ Trigonometria, os árabes contribuíram em três aspectos (MOREY, 2003):

- a introdução de seis funções trigonométricas básicas: seno, cosseno, tangente, cotangente, secante e co-secante;

- a dedução da regra do seno e o estabelecimento de outras identidades;

- a construção de tábuas trigonométricas mais precisas com a ajuda de vários procedimentos de interpolação.

$A b \bar{u}^{\prime} l$-Wafa al Būzjān̄ inventou um método de computar tabelas de senos que fornece o valor do seno

\footnotetext{
${ }^{6} A b \bar{u}^{\prime} l-W a f \bar{a}$ al Būzjōn̄ $(940$ - 998) foi um grande matemático e astrônomo de Bagdá. Contribuiu para a Matemática nos ramos da Geometria e Trigonometria. Ele inventou um método para calcular uma tábua de Senos, que dá a condição de meio grau correto para nove casas decimais (ZELLER 1944).
}

de meio grau correto para nove casas decimais. Segundo Zeller (1944) ele foi, provavelmente, o primeiro a demonstrar a generalidade do teorema do seno relativo a triângulos esféricos. Desenvolveu também as relações para o seno da soma e da diferença de dois arcos desde que seja conhecido o seno de cada um deles:

$$
\operatorname{sen}(a \pm b)=\operatorname{sen} a \cdot \cos b \pm \operatorname{sen} b \cdot \cos a
$$

Ele desenvolveu um estudo especial da tangente e calculou uma tabela das suas linhas. Introduziu a secante e co-secante pela primeira vez e tornou-se conhecedor das relações entre as seis linhas trigonométricas.

O árabe Nasīr al-D̄̄n al-Tūs̄ ficou conhecido por escrever um importante trabalho, O Tratado sobre Quadriláteros, que foi a primeira obra que trata a Trigonometria independente da Astronomia. Segundo Belli (1985) é possível que o trabalho de $a l-T \bar{u} s \bar{\imath}$ tenha chegado às mãos de Regiomontanus na forma de tradução feita no século XII. Já Zeller (1944) é mais incisiva e considera este fato bastante evidente.

O trabalho está dividido em cinco livros que tratam de quadriláteros planos e esféricos. Há aproximadamente 265 figuras ilustrando os cinco livros. Segundo Zeller (1944, p. 9) é difícil dizer se a terminologia no tratado é devido ao autor ou ao tradutor. Ele cita tanto autores árabes quanto gregos. Ao contrário dos escritores medievais, ele dá os devidos créditos aos autores das obras citadas. No seu trabalho há uma grande influência grega, e inúmeros autores gregos são estudados, tais como Euclides e os Elementos, Ptolomeu e o Almagesto, Menelaus e seu tratado sobre Esfera (ZELLER, 1944).

O Tratado sobre Quadriláteros também contém as seis equações fundamentais para a solução de triângulos retângulos esféricos. Mostra como resolver também outros triângulos, substituindo, caso necessário, a consideração dos ângulos pelos lados e reciprocamente, por meio dos triângulos polares 7

Segundo Morey (2003) a lei do seno é atribuída a ele em sua versão moderna, ou seja, dado um triângulo plano qualquer $A B C$ então $\frac{b}{c}=\frac{r \cdot \operatorname{sen} B}{r \cdot \operatorname{sen} C}$ em que $r$ mede 60 unidades.

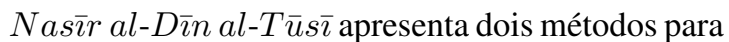
a prova da Lei dos Senos tanto para triângulo agudo

\footnotetext{
7 “Triângulo esférico (euleriano) é a porção da superfície esférica limitada por três arcos de circunferência máxima, menores que $180^{\circ}$. $\mathrm{Ou}$, polígono esférico formado por três lados menores que $180^{\circ}$. Todo triângulo corresponde um triedro com vértice no centro da esfera a qual pertence o triângulo" (ARANA 2006 p. 23). "Dois triângulos esféricos são polares quando os vértices do primeiro são os pólos dos lados homônimos do outro, e reciprocamente" (ARANA 2006 p. 23)
} 


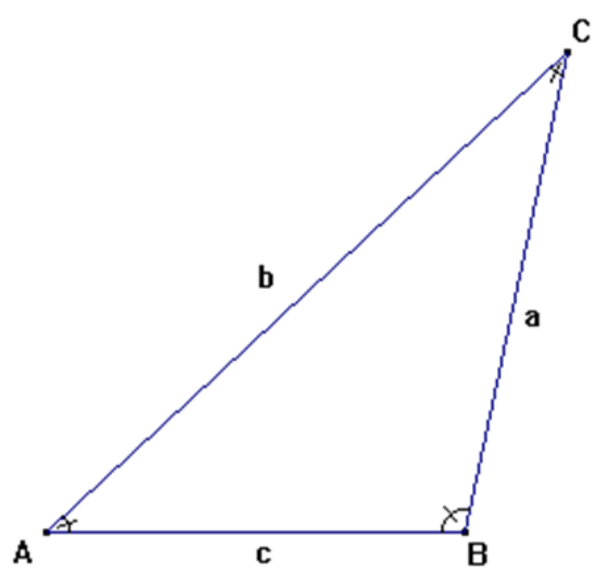

Figura 4: Regra do seno.

Fonte: Elaborada pelas autoras

quanto para obtuso. A primeira prova foi feita pelo

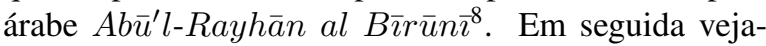
mos a prova de $a l-T \bar{u} s \bar{\imath}$ para a Lei dos Senos (ZELLER 1944, p. 12)

Seja $A B C$, na Figura 5 , os triângulos dados, acutângulo e obtusângulo. Trace $A E$ perpendicular a $B C$. Prolongue $A B$ e $A C$ de modo que $A F=A D=60=$ $R$.

Descreva o arco $D H$. Trace $F K$ e $T D$ perpendicular a $A H$. No triângulo $A B E$, o ângulo $E$ sendo um ângulo reto, $B$ será o complemento de $A ; D T=\operatorname{sen} A$; $A T=\operatorname{sen} B$. No triângulo $A E C, F K=\operatorname{sen} A$; $K A=\operatorname{sen} C$. Devido à semelhança dos dois triângulos $A B E$ e $A D T$, temos

$$
\frac{A B}{A E}=\frac{A D \cdot r}{A T \cdot \operatorname{sen} B} \text { e } \frac{A E}{A C}=\frac{A K \cdot \operatorname{sen} C}{A F \cdot R}
$$

Devido à semelhança dos dois triângulos AEC e AKF, é possível deduzir a afirmação:

$$
\frac{A B}{A C}=\frac{A K \cdot \operatorname{sen} C}{A T \cdot \operatorname{sen} B}
$$

A confecção de tabelas era uma prática corrente na Matemática árabe. Eles compreenderam que tabelas mais precisas facilitavam os cálculos astronômicos. $\mathrm{O}$ árabe Habash al-Hasib computou com precisão a primeira tabela de senos e tangentes de $1^{\circ}$ até a terceira

${ }^{8} A l-B \bar{\imath} r \bar{u} n \bar{\imath}$ escreveu um tratado de Trigonometria intitulado "The key to the knowledge of spherical figures and other figures",

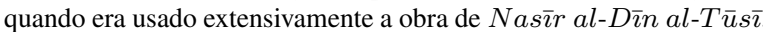
o tratado sobre quadriláteros. Recentes trabalhos científicos árabes creditam a $A b \bar{u}^{\prime} l-R a y h \bar{a} n$ al $B \bar{\imath} r \bar{u} n \bar{\imath}$ a primeira prova da Lei dos Senos para triângulos planos (ZELLER 1944 p. 9). casa sexagesimal, ou seja, até cinco casas decimais. Outros matemáticos trabalharam para reduzir os intervalos e aumentar a precisão destas tábuas. Ulugh Beg (1393 - 1449), por exemplo, computou uma tabela de senos e tangentes com intervalo de $1 \%$, ou seja, $1 \mathrm{mi}-$ nuto, sendo os valores obtidos corretos em pelo menos cinco casas sexagesimais ou nove casas decimais 9

Jamshìd al-Kāsh ${ }^{10}$ (1390-1450) também computou o $\operatorname{sen}\left(1^{\circ}\right)$, aplicando repetidamente a identidade da adição para seno de $3 \alpha$. Substituindo $\alpha=1, A l$ $K \bar{a} \operatorname{shi}$ demonstra que $\mathrm{x}=\operatorname{sen} 1^{\circ}$ é a raiz da equação cúbica

$$
x \equiv \frac{\operatorname{sen} 3^{\circ}+4 x^{3}}{3}
$$

$A l-K \bar{a} s h i$ resolve a equação, usando um equivalente da iteração do ponto fixo, parando na décima casa sexagesimal.

Outros valores de senos e tangente de ângulos, como $12^{\circ}$, que era encontrado pelos árabes, com a aplicação da equação da diferença de dois $\operatorname{arcos} \operatorname{sen}\left(72^{\circ}-60^{\circ}\right)$, uma vez que os senos destes ângulos $\left(72^{\circ}\right.$ e $\left.60^{\circ}\right)$ podiam ser encontrados por meio dos lados de um pentágono regular e de um triângulo equilátero inscrito num círculo.

Utilizando outras equações já conhecidas como a do arco metade, era possível encontrar sen $6^{\circ}, \operatorname{sen} 3^{\circ}, \operatorname{sen}(1$ $1 / 2)^{\circ}$ e $\operatorname{sen}(3 / 4)^{\circ}$

\section{O SURGIMENTO DA TRIGONOMETRIA COMO UMA CIÊNCIA INDEPENDENTE NA EUROPA}

Os séculos de XII a XV foram para a Matemática europeia basicamente um período de assimilação da herança dos matemáticos da Grécia Antiga e do Oriente Hindoarábico. Este período começou com a tradução para o latim, no século XII, de muitas obras clássicas (Euclides, Arquimedes, Al-Khwārizm̄̄ e Al-Ṣābi' Thabit ibn al-Harrān̄̄ Qurra).

Foi nesse período que começaram a se estabelecer as primeiras universidades. A primeira delas foi a de Salerno, na Itália, no século XI; a de Bolonha, na Itália, surgiu no início do século XII. As universidades de Paris e Oxford foram criadas no início do século XII; a de Cambridge, no decorrer do século XIV; as de Praga, Cracóvia, Viena e Heidelberg, no início do século XV,

\footnotetext{
${ }^{9}$ Veja mais detalhes sobre o sen $\left(1^{\circ}\right)$ no artigo de Brummelen (2009).

${ }^{10}$ Astrônomo muçulmano do século XV que propôs a mudança do método geométrico para o algébrico a fim de melhorar a precisão do modelo de Ptolomeu para seno de $1^{\circ}$.
} 


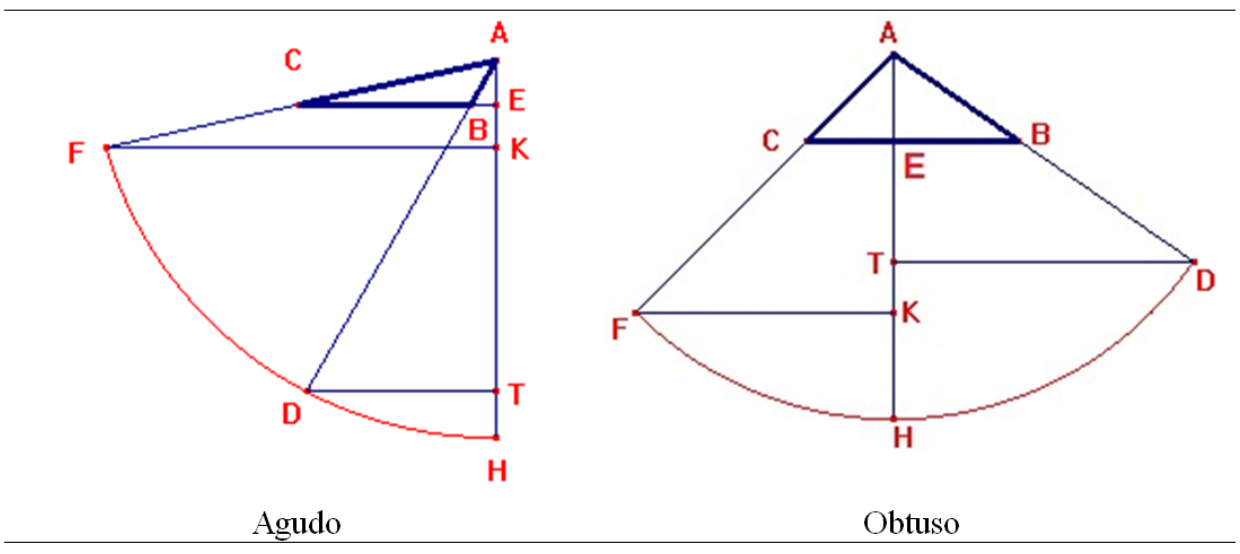

Figura 5: Lei dos Senos para triângulo agudo e obtuso.

Fonte: (ZELLER 1944 p. 9).

entre outros. Convém lembrar que o ensino nessas primeiras universidades se dava por meio do Quadrivium, composto de Aritmética, Geometria, Astronomia e Música.

As disciplinas do Quadrivium eram ministradas por professores de Faculdade de Artes de acordo com a necessidade, sendo que nenhuma universidade da Europa preparava professores especificamente de Matemática. Um dos primeiros a se especializar no ensino de disciplinas matemáticas foi Johann Gmunden ${ }^{11}$ mestre na Universidade de Viena. Com ele foi iniciada a tradição que colocou a Universidade de Viena entre as melhores no ensino de Matemática na Europa. Nesse período nasceu Johann Müller Regiomontanus que teve um papel importante para a história da trigonometria moderna 12

Ligado a Astronomia, Regiomontanus, atacou vários problemas discutidos no século XV e XVI, dentre eles podemos citar a sistematização e a generalização dos métodos de medição de grandezas geométricas por meio da Trigonometria Plana e Esférica, o aperfeiçoamento dos métodos e instrumento de cálculo, a elaboração de tabelas matemáticas no sistema decimal, assim como a transformação da Álgebra retórica para a simbólica (BELLI, 1985). Porém, muitas se suas obras, tais como De Triangulis Omnimodis Libri Quinque (1533), Tabulae directionum et profectionum (1490), Tabulae primi mobilis ( 1468), estava diretamente lidados a Matemática e principalmente a Trigonometria.

\footnotetext{
${ }^{11}$ Johann Gmunden ( 1380 - 1442) foi um matemático e astrônomo austríaco. Lecionou na Universidade de Viena em que seu sucessor foi Peuerbach. Escreveu sobre tabelas planetárias e Calendários. O asteróide Johannesgmunden foi nomeado em sua honra.

${ }^{12}$ Mais detalhes em Pereira (2010)
}

Em seu tratado De Triangulis Omnimodis Libri Quinque composto por cinco livros no total de 131 páginas, ele sistematiza todos os conceitos de Trigonometria Plana e Esférica, trazendo uma linguagem atual, acarretando seu uso por diversos astronomos posteriorer, tais como: Nicolaus Copérnico (Polônia: 1473-1543), Georg Joachim von Lauchen Réticos (Áustria: 1514 1574) e Pedro Nunes (Portugal: 1502 - 1578).

Conceitos de seno, lei dos senos, lei dos cossenos, entre outros, são largamente citados na obra De Triangulis, fazendo um tratamento de aplicação voltado para a Astronomia. Um exemplo dessa linguagem é o conceito de seno.

O seno, na época de Regiomontanus, difere ligeiramente da definição da função seno de hoje. O seno, como usado em sua obra, é uma perpendicular traçada de uma extremidade de um arco de um círculo para o diâmetro que foi traçado pela outra extremidade do arco. O seno reverso é a parte do diâmetro entre o pé daquela perpendicular ou o seno e o arco. O seno do complemento do arco é o seno da diferença entre o arco e um quadrante; consequentemente, se o arco é menor que $90^{\circ}$, o complemento do arco é $90^{\circ}$ menos os graus do arco, mas, se o arco é maior que $90^{\circ}$, o complemento é levado a ser os graus do arco menos $90^{\circ}$. Vale ressaltar que o teorema 20 do livro I mostra a definição do seno:

Teorema 20: Em todo triângulo retângulo, um dos quais o vértice agudo é o centro de um círculo e cuja [hipotenusa] é seu raio, o lado que subtende a este ângulo agudo é o seno reto do arco adjacente ao lado oposto ao ângulo dado, e o terceiro lado do triângulo é igual ao seno do complemento do arco.

Esquematizando... Se o triângulo retângulo $\triangle A B C$ é dado com $C$ o ângulo reto e $A$ um ângulo agudo, em torno do vértice do qual o círculo $B E D$ é descrito com 
a hipotenusa - que é, o lado oposto ao maior ângulo - com o raio, e se o lado $A C$ é estendido suficientemente para encontrar-se com a circunferência do círculo no ponto $E$, então o lado $B C$ oposto ao $\angle B A C$ é o seno do arco $B E$ que subtende ao ângulo dado, e além disso o terceiro lado $A C$ é igual ao seno reto do complemento arco $B E$.

Demonstração Estenda o lado $B C$ para encontrar a circunferência do círculo no ponto $D$. Do ponto $A$, o centro do círculo, trace um raio $A K$ paralelo ao lado $B C$, e do ponto $B$ trace uma corda $B H$ paralela ao lado $A C$. As duas linhas $B H$ e $A K$ necessariamente se interceptam, devido os ângulos $A B H$ e $B A K$ serem agudos, e isso acontece no ponto $G$. Consequentemente, como o raio $A E$ intercepta a corda $B D$ perpendicularmente devido o $\angle A C B$ reto, $[A E]$ bissecta ambos a corda $B D$, de acordo com o Teorema 3 do livro III dos Elementos de Euclides, e o arco $B D$, de acordo com a Teorema 29 do livro III dos elementos de Euclides. Assim, apenas pela definição a linha inteira $B D$ é a corda do arco $B D$, então também [pela definição] sua metade, isto é a linha $B C$, é o seno do arco-metade $B E$ oposto ao $\angle B A E$, ou $B A C$. E este é o que a primeira parte do teorema afirma.

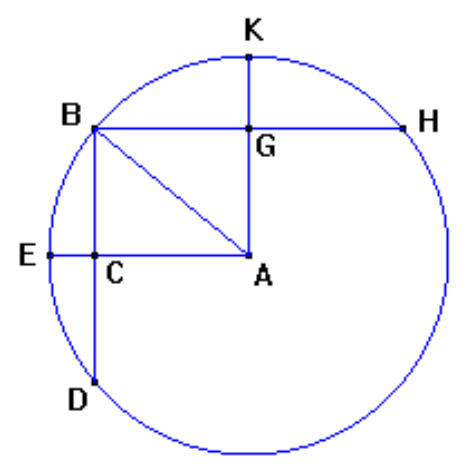

A segunda parte é mostrada para ser verdade se for primeiro compreendido que, pela Proposição 34 do Livro I dos Elementos de Euclides, o $\angle A G B$ é um ângulo reto, então o raio $A K$ bifurca a corda $B H$ e seu arco, como mencionado acima. Consequentemente, através da definição, a linha reta $B G$ é o seno de arco $B K$. Além disso, a linha $B G$ é igual ao lado [ $A C]$ do $\triangle A B C$ pela Proposição 34 do Livro I dos Elementos de Euclides devido à área de $A G B C$ ser limitada por linhas paralelas. Mas o $\angle C A G$, ou $E A K$, é um ângulo reto pela Proposição 29 do livro I dos Elementos de Euclides, devido à linha $B C$ ser paralela a $A G$. Consequentemente pela última Proposição do Livro VI dos Elementos de Euclides, o arco $E K$ é mostrado para ser um quadrante da circunferência. Consequentemente, o arco $B K$ está definido como o complemento do arco $B E$, e o seno BG [do arco $B K$ ] há pouco foi mostrado como sendo igual ao lado $A C$. E assim ambas as partes do teorema foram provadas. REGIOMONTANUS; HUGHES 1967 p. 58 - tradução nossa) - Grifo nosso.

No Teorema 20, Regiomontanus utiliza a definição apresentada no início do Livro I: Quando o arco e a corda são bissectados, nós chamamos aquela meiacorda de seno reto do arco metade (REGIOMONTA-
NUS; HUGHES, 1967, p. 30-31) para embasar a demonstração de seu teorema. Ele toma o raio do círculo trigonométrico igual a 1 , e ou seja, definindo o $\operatorname{sen} B \hat{\mathrm{A}} C=\overline{B C}$. Ele ainda utiliza o termo "seno reto do complemento do arco $B E$ ", em que se refere ao cosseno do ângulo $B \hat{\mathrm{A}} C$, sendo $\cos B \hat{\mathrm{A}} C=\overline{A C}$

\section{CONSIDERAÇÕES FINAIS}

A trigonometria, ciência que é estudada na Educação Básica e Superior, pouco é apresentada aos estudantes conceitos oriundos de seu desenvolvimento histórico. Muito do que é apresentada nos atuais livros didáticos só se remete ao estudo de funções circulares, ou seja, somente cerca de cinco séculos de história.

Outro ponto é o descaso dos currículos escolares em relação à Trigonometria Esférica. Ela, conhecida em alguns cursos do Ensino Superior, e desconhecida para os alunos do Ensino Fundamental e Médio, atualmente, está atrelada a disciplina básica dos cursos técnicos ou das engenharias ligadas a: Cartografia, Astronomia, entre outros.

No curso de Bacharelado em Matemática no Brasil, uma disciplina que aborda a Trigonometria Esférica é a Geometria não-Euclidiana, no estudo da Geometria Elíptica. Porém, na formação do professor de Matemática, ou seja, nos cursos de Licenciaturas, numa pesquisa realizada em 2005, por Barreto e Tavares (2007. p. 2) das 47 Instituições de Ensino Superior brasileiras pesquisadas por eles, somente cinco abordavam conceitos de Geometrias não-Euclidianas nas suas matrizes curriculares, consequentemente o estudo de triângulos esféricos. Isso nos leva a concluir que a Trigonometria Esférica está perdendo lugar no ensino de Matemática.

Desse modo, consideramos é necessário uma abordagem diferenciada da história da trigonometria, não remontando a fatos, datas e biografias, mas envolvendo conceitos trigonométricos desenvolvidos ao longo da história que ainda são tratados em sala de aula.

\section{REFERÊNCIAS}

ARANA, J. M. Trigonometria Esférica: Notas de aula. Presidente Prudente: [s.n.], 2006.

ASGER, A. Episódios da história antiga da matemática. Rio de Janeiro - SBM, 1984.

BARRETO, M. d. S.; TAVARES, S. Do mito da geometria euclidiana ao ensino das geometrias não euclidianas. Vértices, Campos dos Goytacazes, v. 9, n. 1/3, jan./dez. 2007. 
BELLI, I. A. Johann Muller (Regiomontanus) 1436-1476. Moscou: Naúka: Courier Corporation, 1985.

BRUMMELEN, G. V. The mathematics of the heavens and the Earth: the early history of trigonometry. New Jersey: Princeton University Press, 2009.

KENNEDY, E. S. História da trigonometria. São Paulo: Atual Editora, 1992. Tradução de Hygino H. Domingues.

MOREY, B. Geometria e trigonometria na Índia e nos países árabes. Editora SBHMat, Rio Claro, 2003. Coleção História da Matemática para Professores.

MOREY, B. B. Tópicos de história da trigonometria. Natal: SBHMat, 2001. Coleção História da Matemática para Professores.

NEUGEBAUER, O. E. The exact sciences in antiquity. New York: Dover Publication, 1969. v. 9.

PEREIRA, A. C. C. A obra "de Triangulis omnimodis libri quinque” de Johann Müller Regiomontanus (1436-1476): uma contribuição para o desenvolvimento da trigonometria. Tese (doutorado em Educação) — Universidade Federal do Rio Grande do Norte, Natal, 2010. 329 f.

REGIOMONTANUS; HUGHES, B. Regiomontanus on triangles. [S.1.]: University of Wisconsin Press, 1967.

ZELLER, M. C. The Development of Trigonometry from Regiomontanus to Pitiscus. Tese (doutorado) University of Michigan, Ann Arbor, 1944.

ZINNER, E. Regiomontanus, his life and work. Borth-Holland: [s.n.], 1990. 